\author{
Erratum
}

\title{
Nanoimprint lithographic surface patterning of sol-gel fabricated nickel ferrite (NiFe2O4) - ERRATUM
}

Goran Rasic and Justin Schwartz

doi: 10.1557/mrc.2013.44, Published by Materials Research Society with Cambridge University Press, 11 November 2013.

In Rasic et al. ${ }^{1}$, the miller indices are displayed incorrectly in two instances:

On page 209, first column, fifth line, (111) direction should be $<111>$ direction.

On page 209, second column, seventh line, $<111>$ family should be $\{111\}$ family.

In addition, on page 209, first column, first line, Volmer-Webber should be spelled Volmer-Weber.

The publisher regrets the mistakes.

\section{Reference}

1. Goran Rasic and Justin Schwartz: Nanoimprint lithographic surface patterning of sol-gel fabricated nickel ferrite (NiFe204). MRS Communications 3(4), 207-211 (2013). 